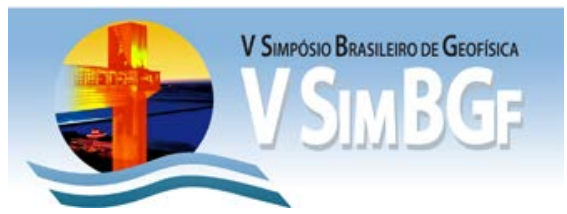

\section{Improved seismic reprocessing with enhanced PSTM image for offshore Campos basin area - A case history}

L. J. Fei, G. Mengqiu, L. Jun (Landocean Energy Service

Co. Ltd)

M. A. Almeida, C. S. Goulart (OGX Petróleo e Gás)

Copyright 2012, SBGf - Sociedade Brasileira de Geofísica

Este texto foi preparado para a apresentação no V Simpósio Brasileiro de Geofísica, Salvador, 27 a 29 de novembro de 2012. Seu conteúdo foi revisado pelo Comitê Técnico do V SimBGf, mas não necessariamente representa a opinião da SBGf ou de seus associados. É proibida a reprodução total ou parcial deste material para propósitos comerciais sem previa autorização da SBGf.

\section{Summary}

Focusing on the characteristics of marine seismic data, especially the geophysical challenge caused by poor imaging of covering carbonate layer, growing multiples, weak reflections, and low $\mathrm{S} / \mathrm{N}$ in the study area from Campos basin, the processing sequence was designed accordingly as a comprehensive complementation with key technologies of pre-stack noise attenuation, prestack amplitude compensation, predictive deconvolution, CMP-based high precision Radon transform interbed attenuation, anisotropy curved tray Pre-stack time Migration(PSTM) etc.. The final result showed the enhanced structure geometry of sand and carbonate reserve with clear fault block and break point in target zone, which supplied a good solution for imaging challenge in the area.

\section{Introduction}

Campos Basin is one of the twelve eastern Brazilian marginal basins, which lie beneath the coastal plain, continental shelf and slope of the western portion of the South Atlantic Ocean. Their tectonic and sedimentary evolution is linked to the Neocomian breakup of Gondwana, and the subsequent opening of the South Atlantic Ocean.

Because of the complicated geological condition in target layer in study area, the major problems left in previously processed results are poor structural imaging, insufficient fidelity which will degrade the interpretation precision(As shown in Figure (1)

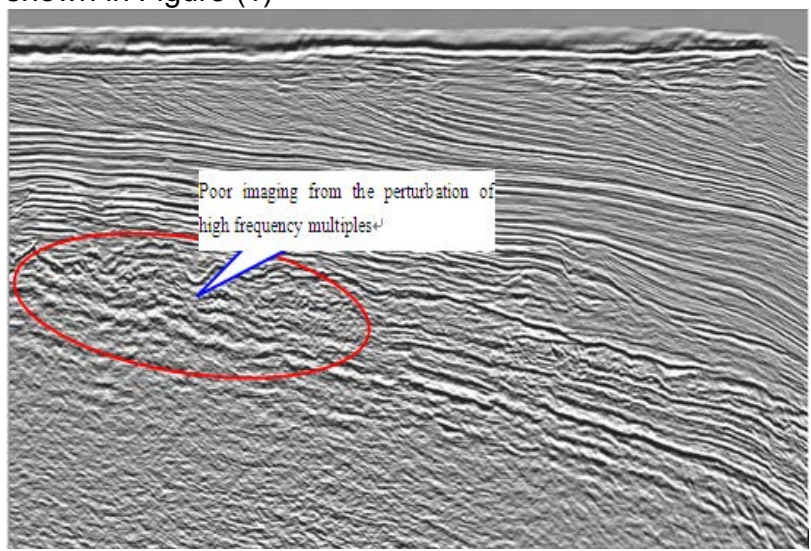

Figure 1-PSTM section from previous processing (Poor image from the perturbation of high frequency multiples)

\section{Processing challenges and strategies}

Regarding the problems left in previous processing, for the reprocessing project the decision was taken to evaluate the advance of a well organized comprehensive processing sequence. These advances may be categorized into three main areas: resolution improving, multiple attenuation and time-imaging.

(1) Poor image quality, narrow effective frequency band, and unclear wave group characteristics in mid-to-deep section.

Solution: multi-channel predictive deconvolution was adopted to compress the wavelet, strengthen the signal from dominant frequency band in target layer, enhance the wave group characters and improve the $\mathrm{S} / \mathrm{N}$ ratio.

(2) Strong interference from interbed multiples

Solution: Based on the better conditioned CMP gathers after deconvolution, the high-precision Radon transform demultiple method was used to enhance the effective reflections in pre-stack gathers.

(3) Low S/N and complex structure make it difficult for accurate imaging

Solution: An accurate velocity field is built by multiiterations and anisotropy curved ray PSTM to improve the final image. The velocity field is optimized by velocity scan and used to collapse the diffractions, move the reflections to correct positions and obtain a final accurate image.

\section{Key technologies}

(1)Multi-channel predictive deconvolution:

Predictive deconvolution can compress wavelet to achieve a high resolution. It can also suppress the noises like multiples and singing (Robinson and Treitel, 1980). Review of the raw data showed abundantly growing noises including short period multiples, random noised and low frequency noises. By using predictive deconvolution, wavelet was compressed and part of interbed multiples were simultaneously suppressed. A good result was obtained by using parameters got from parameter testing of predictive distance and application window, the dominant frequency component of reflections was enhanced, and the wave group character were improved(See Figure 2) 


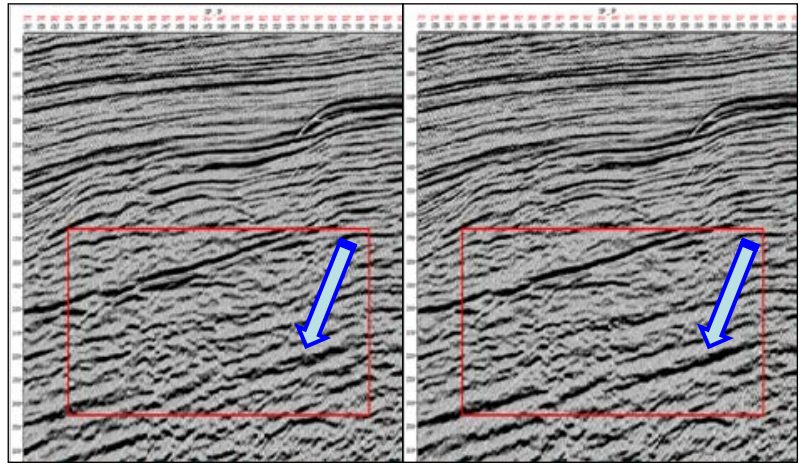

Figure 2 -Enlarged section before (left) and after (right) deconvolution

(2) Radon transform multiple attenuation technology: multiples are mainly growing in North-West part of the area, with long path multiples dominated and with high frequency. Multiple attenuation can be achieved by the Radon transform, using velocity discrimination of multiple reflections with respect to primary reflections. The Radon transform is defined as an integration of data along certain stacking paths (Radon, 1917). The basic concept of Radon transform de-multiple method is as below (Cary, 1998);

$$
\begin{aligned}
& v(q, \tau)=\sum u\left(x, t=\tau+q x^{2}\right) \\
& u(x, t)=\sum u\left(q, \tau=t-q x^{2}\right)
\end{aligned}
$$

Here, $q$ is the curvature of parabola centered at zero offset, $\tau$ is the intercept time. When we get an accurate velocity of primaries, $q$ of positive curvatures are multiples and should be picked out.

The way to suppress multiples In $(\tau, p)$ domain by moveout difference between primaries and multiples is, perform $\tau$ - $p$ transform on CMP gathers with NMO by using the primary velocity, then filtering in $(\tau, p)$ domain, cut the data with $p>0$, and then perform $\tau$ - $p$ reverse transform, the output will be the data with multiple eliminated. It was proved that, after apply NMO on gathers by using the primary velocity, multiples will drop in a section with $p>0$ in $\tau$-p domain.

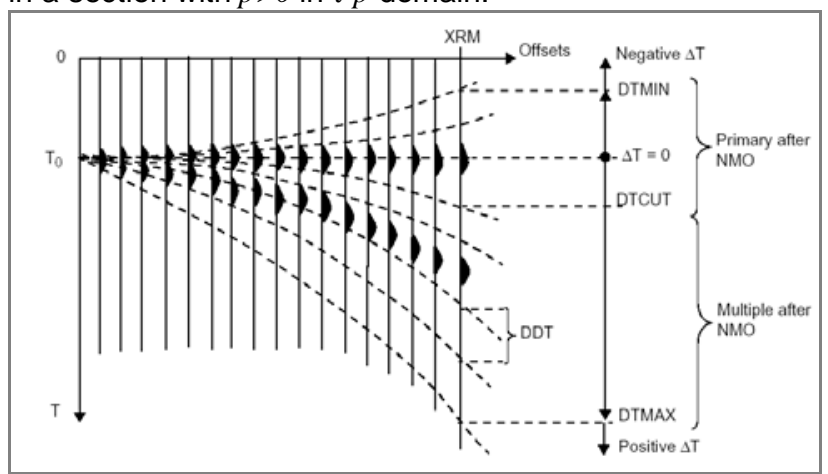

Figure 3 -Model of Radon transform multiple attenuation(Yilmaz,2001; XRM is the maximum offset, $D T M I N$ is the minimum range for multiple suppression, DTCUT is the start time below the horizontal line, DDT is the scanned parabolic increasing, DTMAX is the maximum time of multiple attenuation. The shadowed part is the area with multiples growing in $\tau-P$ domain, if the primary velocity is accurate enough, primary reflection and multiple will be separated in $\tau-P$ domain)

In this study, multiples were removed in two steps:

On the first hand attenuate multiples with large velocity difference from primaries at far-offset part of CMP gathers, and secondly attenuate the near-offset multiples from CRP gathers on which the faults has been moved to correct position by migration

An accurate velocity field was obtained by multi-iterations of velocity picking after deconvolution and used to attenuate multiples on CMP gathers. Effective reflections were further strengthened (See Figure 4)

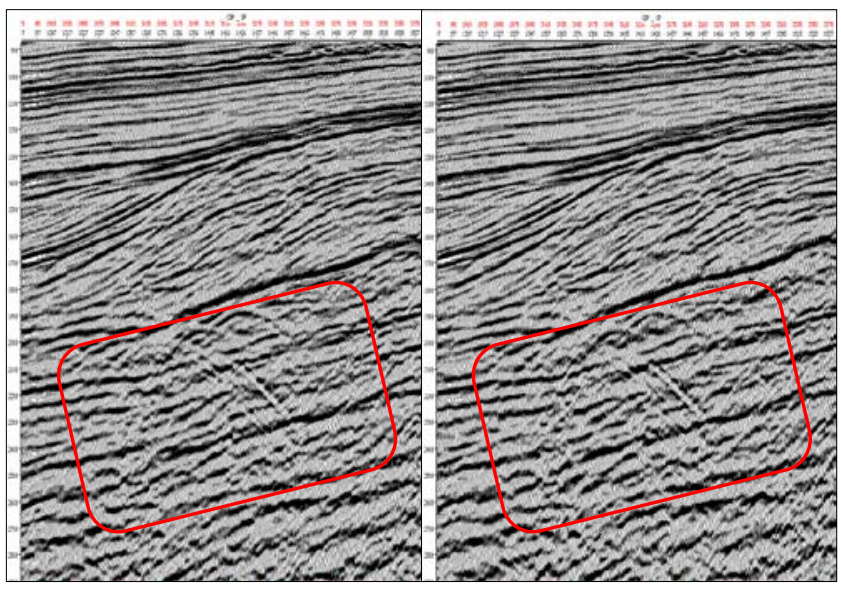

Figure 4 -Enlarged section before (left) and after (right) multiple attenuation

(3) Anisotropy curved ray pre-stack time migration:

Isotropic PSTM is not enough to sculpture the image of complicated subsurface structure. PSTM with Anisotropy taken into consideration supplies a solution for complex structure imaging with being more clear and accurate. In sedimentary basin, the most common anisotropic problem is caused by isotropic media with vertical asymmetrical (VTI). Thomsen (1986) proposed that the wave field in a homogeneous and week anisotropy media can be described by vertical velocity, $V_{P 0}$ and $\varepsilon, \delta$.

To do anisotropy PSTM, two key parameters are needed, ${ }_{P 0}$, which is the vertical velocity; and $\eta$, which is the anellipticity.

$$
\eta=\frac{(\varepsilon-\delta)}{1+2 \delta}
$$

$\eta$ can be obtained by extend the muting line to offset extent which larger than target depth after preserving data of the near offset (smaller than target depth) .

After anisotropy PSTM, both reflections of near or far offset have been flattened, and the image accuracy has been improved by enhance the $\mathrm{S} / \mathrm{N}$ and energy of reflections.

The equation for anistropy Kirchhoff PSTM is, 
$u\left(x_{p}, y_{p}, z_{p}, t\right)=\frac{1}{2 \pi} \iint_{\theta_{0}} \cos \varphi \frac{1}{v r} \frac{\partial}{\partial t} u\left(x, y, 0, t+\frac{r}{v}\right) d \theta$

$\theta$ : The closure surface of the turbulence area $r$ : the outer normal of $\theta$;

$r=p(x p, y p, z p)$ : the distance to each points on $\theta$;

$u(x p, y p, z p, t)$ : wave field function of the point $\mathrm{P}(x p, y p$,

$z p)$ on $\theta$ at time $\mathrm{T}$;

The final stacking imaging was improved gradually by fine velocity iterations. The stacking velocity can be used as reference velocity for velocity scan, by combining with anisotropy velocity analysis, to create the PSTM velocity field and use it for migration. Final PSTM result can be seen in Figure 5, which showed collapsed diffraction, correctly migrated subsurface reflections and reasonable structure image.

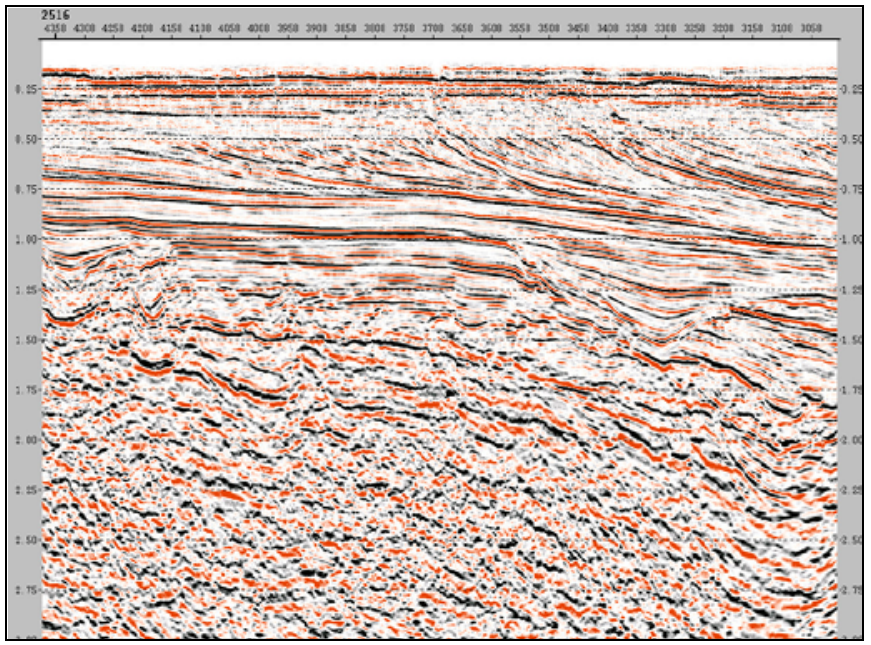

Figure 5 -PSTM section (before denoising)

\section{Results obtained and Conclusions}

This reprocessing study was considered a success. It showed that the implementation of an up to date preprocessing flow can greatly improve the image quality. Based on a thoroughly analysis of seismic data, a comprehensive usage of multi-channel predictive deconvolution, fine velocity analysis and iterations, data regularization, Radon transform de-multiple in both CMP and CRP domain, and curved ray PSTM has been performed, which supplies a good processing solution in study area, final result showed considerable improvement when it was compared to the previously processed result. It approached the goal of fine structural interpretation (Figure5, Figure6):

1. High resolution in shallow-middle section, clear unconformity contact relationship, and a significantly raised S/N, See 300-1300ms in Figure 6 (b)

2. Clear image of structure in target layer and obvious wave group character showed after efficient multiple attenuation (See Figure 6), especially the image of carbonate layer in 1300-2500m, which achieved an improved accuracy, and will benefit future interpretation and reservoir prediction.

3. Correct shift of reflections to their right positions, clear fault image and correct combinational relationship of fracture system;

\section{ACKNOWLEDGMENT}

The authors are grateful to Jose Luiz Flores, Davilson Eduardo Andrade and Flavio Burda for valuable discussions and support and also kindly thank to OGX for granting permission to publish the data.

\section{References}

Cary, P., 1998, The simplest discrete Radon transform: Presented at the 68th Ann. Internat. Mtg., Soc. Expl. Geophys., Expanded Abstracts, 1999-2002.

Radon J 1917, U“ ber die Bestimmung von Funktionnen durch ihre Integralwerte la"ngs gewisserMannigfaltikeiten Ber. Verh. Sachs. Akad. Wiss. Leipzig-Math.-Natur. KI. 69 262-77

Robinson, E. A., 1980, Physical applications of stationary time-series-With special reference to digital data processing of seismic signals: Macmillan

Thompson, L., 1986. Weak elastic anisotropy. Geophys., 51, 1954-1966.

Yilmaz, Ö. 2001. Seismic data analysis. Society of Exploration Geophysicists 


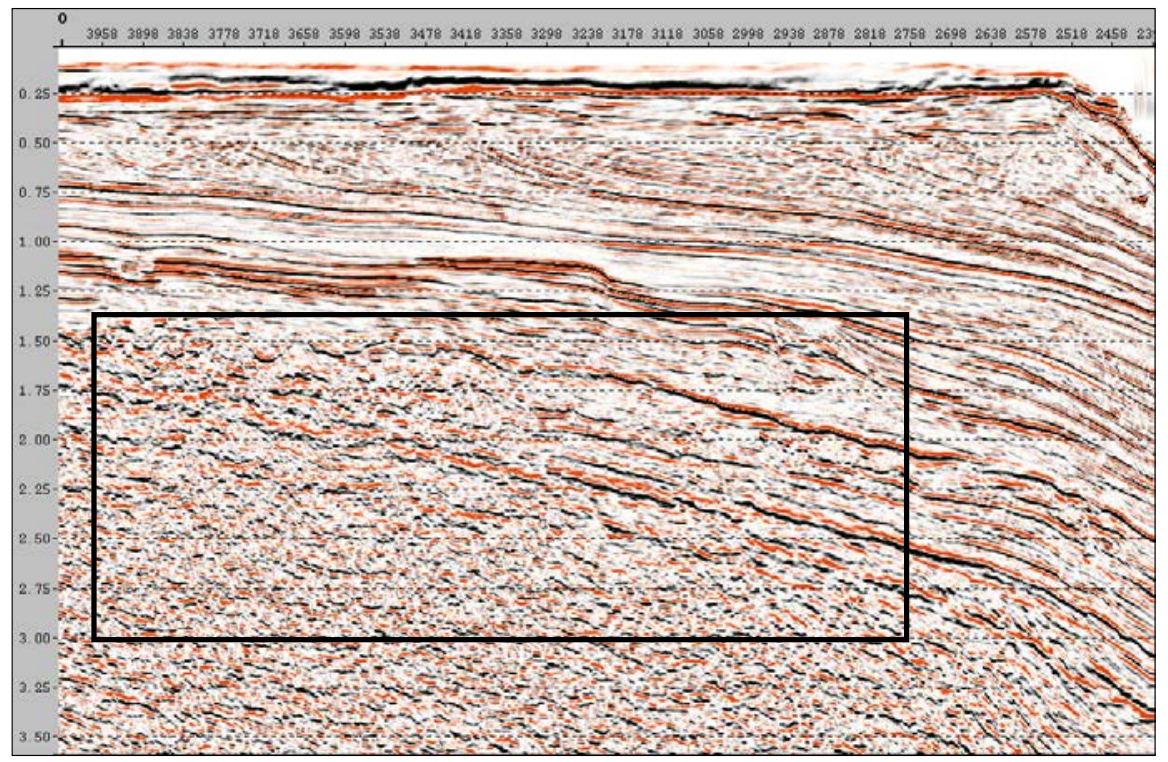

Figure 6a -PSTM result from previous processing

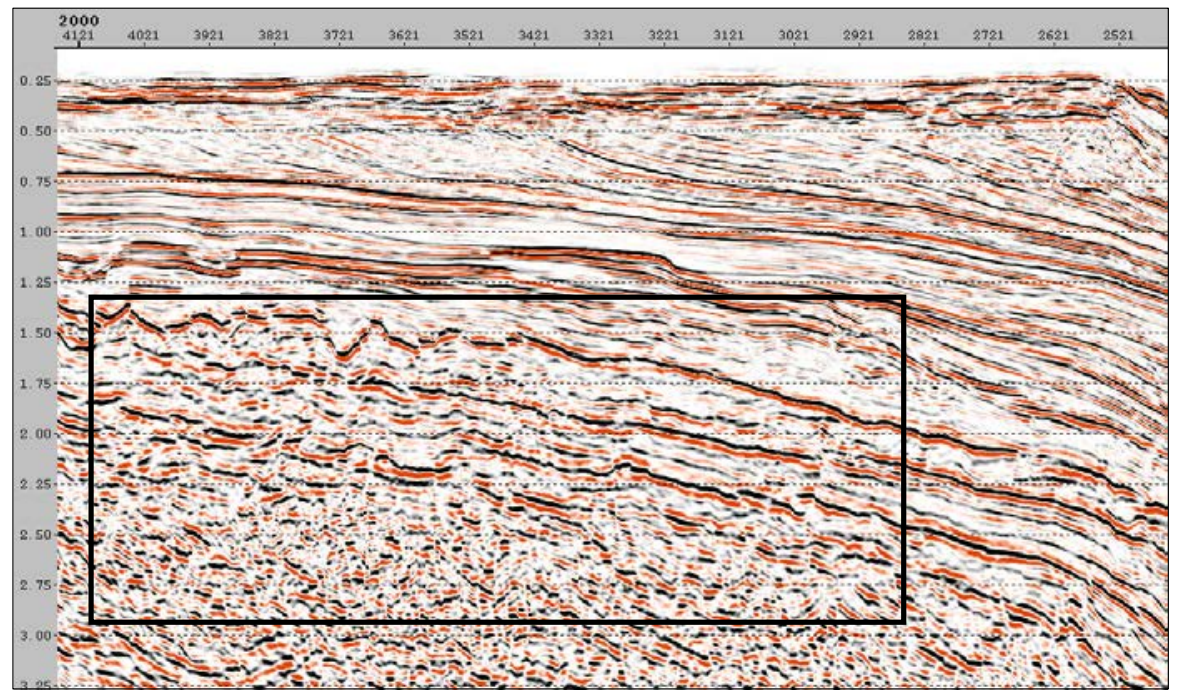

Figure $6 b$ - New PSTM result 